\title{
Allenyl and Alkynyl Selenols and Selenocyanates. Synthesis, Spectroscopic Characterization and Quantum Chemical Study
}

\author{
Jean-Claude Guillemin, ${ }^{*}{ }^{\dagger}$ Gábor Bajor, ${ }^{\S}$ El Hassan Riague, ${ }^{\dagger}$ Brahim Khater, ${ }^{\dagger}$ Tamás \\ Veszprémi* $^{*} \S$ \\ † Sciences Chimiques de Rennes, UMR 6226 CNRS-ENSCR, Ecole Nationale Supérieure de \\ Chimie de Rennes, 35700 Rennes, France, and ${ }^{\S}$ Budapest University of Technology and \\ Economics 1521 Budapest, Gellert ter 4, Hungary.
}

\section{Supporting Information}

Tables $1 \mathrm{~S}$ and 2S, ${ }^{1} \mathrm{H}$ and ${ }^{13} \mathrm{C}$ NMR spectra of compounds 6, 9-12 and 14. This material is available free of charge via the Internet at http://pubs.acs.org.

To whom correspondence should be addressed. E-mail : jean-claude.guillemin@enscrennes.fr, Fax: (+33) 2232381 08, and E-mail : Tveszpremi@mail.bme.hu, Fax: (+361) 4633642 
Table 1S.

Calculated bond lengths of alkynyl selenols and selenocyanates [in $\AA$ ]*.

\begin{tabular}{|c|c|c|c|c|c|c|c|c|c|}
\hline \multirow{2}{*}{\multicolumn{2}{|c|}{ Molecule }} & \multirow{3}{*}{$\begin{array}{c}\begin{array}{c}\text { Confor- } \\
\text { mation }\end{array} \\
1\end{array}$} & \multicolumn{7}{|c|}{ Geometrical Parameter } \\
\hline & & & $\mathbf{a}$ & $\mathbf{b}$ & $\mathbf{C}$ & d & $\mathbf{e}$ & $\mathbf{f}$ & g \\
\hline & ${ }^{b}$ Se $\frac{\text { e }}{-} \mathbf{H}$ & & $1.217(1.202)$ & $1.824(1.838)$ & & & $1.454(1.476)$ & & \\
\hline & 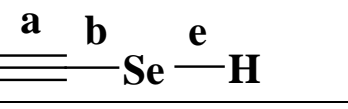 & 2 & $1.220(1.205)$ & $1.824(1.838)$ & $1.457(1.454)$ & & $1.454(1.476)$ & & \\
\hline 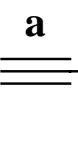 & $\overbrace{\mathrm{Se}}^{\mathrm{c}} \stackrel{\mathrm{e}}{\mathrm{H}}_{\mathbf{H}}$ & $\begin{array}{l}3 \mathbf{a} \\
3 \mathbf{b}\end{array}$ & $\begin{array}{l}1.215(1.199) \\
1.215(1.200)\end{array}$ & $\begin{array}{l}1.961(2.003) \\
1.959(1.999)\end{array}$ & $\begin{array}{l}1.451(1.444) \\
1.449(1.443)\end{array}$ & & $\begin{array}{l}1.453(1.474) \\
1.452(1.471)\end{array}$ & & \\
\hline a & ${ }^{b} \mathrm{Se} \frac{\mathrm{e}}{-} \mathrm{H}$ & $\begin{array}{l}4 a \\
4 b \\
4 c \\
4 d \\
4 e \\
\end{array}$ & $\begin{array}{l}1.215(1.199) \\
1.215(1.199) \\
1.215(1.199) \\
1.215(1.200) \\
1.215(1.199) \\
\end{array}$ & $\begin{array}{l}1.944(1.978) \\
1.955(1.991) \\
1.945(1.980) \\
1.945(1.978) \\
1.951(1.987) \\
\end{array}$ & $\begin{array}{l}1.459(1.458) \\
1.459(1.459) \\
1.458(1.457) \\
1.460(1.460) \\
1.459(1.458) \\
\end{array}$ & $\begin{array}{l}1.528(1.533) \\
1.527(1.531) \\
1.528(1.533) \\
1.527(1.533) \\
1.527(1.532) \\
\end{array}$ & $\begin{array}{l}1.453(1.472) \\
1.452(1.472) \\
1.453(1.473) \\
1.453(1.473) \\
1.452(1.472) \\
\end{array}$ & & \\
\hline & ${ }^{b} \mathrm{Se} \stackrel{\mathrm{f}}{-} \mathrm{C} \stackrel{\mathrm{g}}{\equiv} \mathrm{N}$ & 5 & $1.217(1.201)$ & $1.822(1.836)$ & & & & $1.834(1.856)$ & $1.176(1.154)$ \\
\hline & 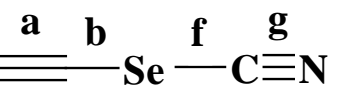 & 6 & $1.219(1.204)$ & $1.819(1.834)$ & $1.455(1.452)$ & & & $1.835(1.858)$ & $1.176(1.155)$ \\
\hline$\underline{\underline{\mathbf{a}}}$ & $\overbrace{\mathrm{Se}}^{\mathrm{b}} \frac{\mathrm{f}}{\mathrm{C}} \mathrm{g} \stackrel{\mathrm{g}}{\mathrm{N}}_{\mathrm{N}}$ & $\begin{array}{l}7 \mathbf{a} \\
7 \mathbf{b}\end{array}$ & $\begin{array}{l}1.215(1.199) \\
1.215(1.199)\end{array}$ & $\begin{array}{l}1.967(2.015) \\
1.966(2.011)\end{array}$ & $\begin{array}{l}1.448(1.441) \\
1.445(1.439)\end{array}$ & & & $\begin{array}{l}1.827(1.845) \\
1.824(1.843)\end{array}$ & $\begin{array}{l}1.177(1.156) \\
1.177(1.156)\end{array}$ \\
\hline & $\stackrel{d}{b}_{S e} \frac{f}{C} \stackrel{g}{\equiv} N$ & $\begin{array}{l}8 a \\
8 b \\
8 c \\
8 d \\
8 e \\
\end{array}$ & $\begin{array}{l}1.214(1.198) \\
1.215(1.199) \\
1.215(1.199) \\
1.215(1.199) \\
1.214(1.199)\end{array}$ & $\begin{array}{l}1.952(1.991) \\
1.960(1.999) \\
1.951(1.988) \\
1.951(1.986) \\
1.955(1.994)\end{array}$ & $\begin{array}{l}1.457(1.456) \\
1.459(1.459) \\
1.457(1.457) \\
1.459(1.459) \\
1.458(1.458)\end{array}$ & $\begin{array}{l}1.526(1.530) \\
1.526(1.529) \\
1.525(1.530) \\
1.525(1.531) \\
1.527(1.532)\end{array}$ & & $\begin{array}{l}1.825(1.844) \\
1.827(1.845) \\
1.825(1.845) \\
1.825(1.845) \\
1.824(1.843)\end{array}$ & $\begin{array}{l}1.177(1.155) \\
1.177(1.156) \\
1.177(1.156) \\
1.177(1.156) \\
1.177(1.156)\end{array}$ \\
\hline
\end{tabular}

* MP2/cc-pVTZ calculations. Data of B3LYP/cc-pVTZ level are in bracket. 
Table 2S.

Calculated bond lengths of allenyl selenols and selenocyanates [in $\AA$ ]*.

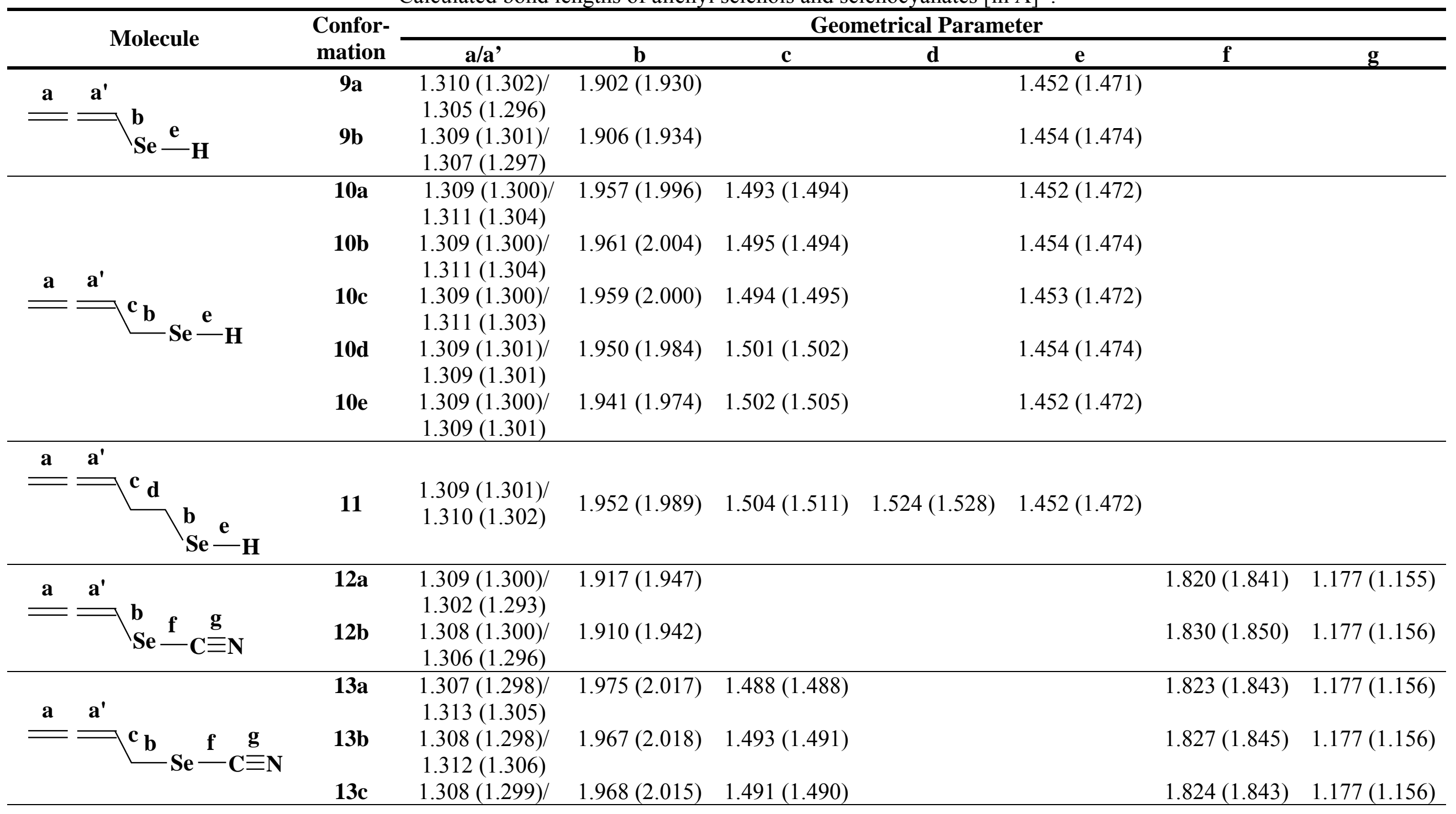


$1.311(1.304)$

13d $1.310(1.301) /$

$1.954(1.991) \quad 1.500(1.501)$

$1.829(1.847) \quad 1.177(1.156)$

13e $\quad 1.308(1.299)$

$1.308(1.299) / \quad 1.944(1.981) \quad 1.501(1.503)$

$1.825(1.845) \quad 1.177(1.156)$ $1.309(1.301)$

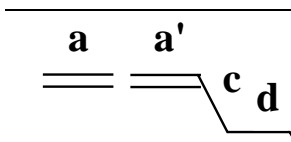

C d

$\mathrm{Se}_{\mathbf{S}} \stackrel{\mathbf{f}}{\mathrm{g}} \mathbf{\equiv} \mathbf{N}$

$1.956(1.995) \quad 1.505(1.507) \quad 1.524(1.527)$

$1.824(1.847) \quad 1.177(1.156)$

* MP2/cc-pVTZ calculations. Data of B3LYP/cc-pVTZ level are in bracket. 

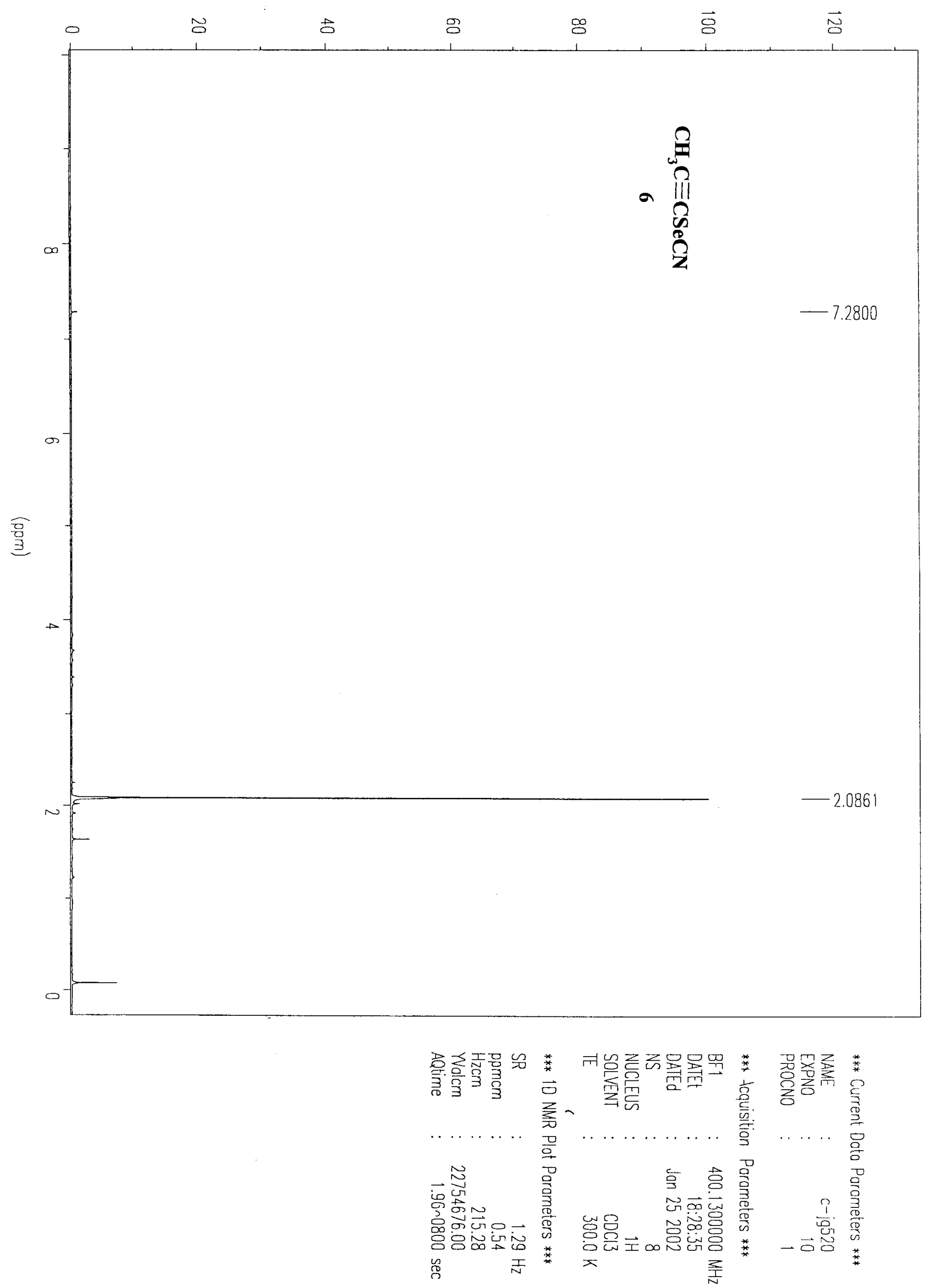

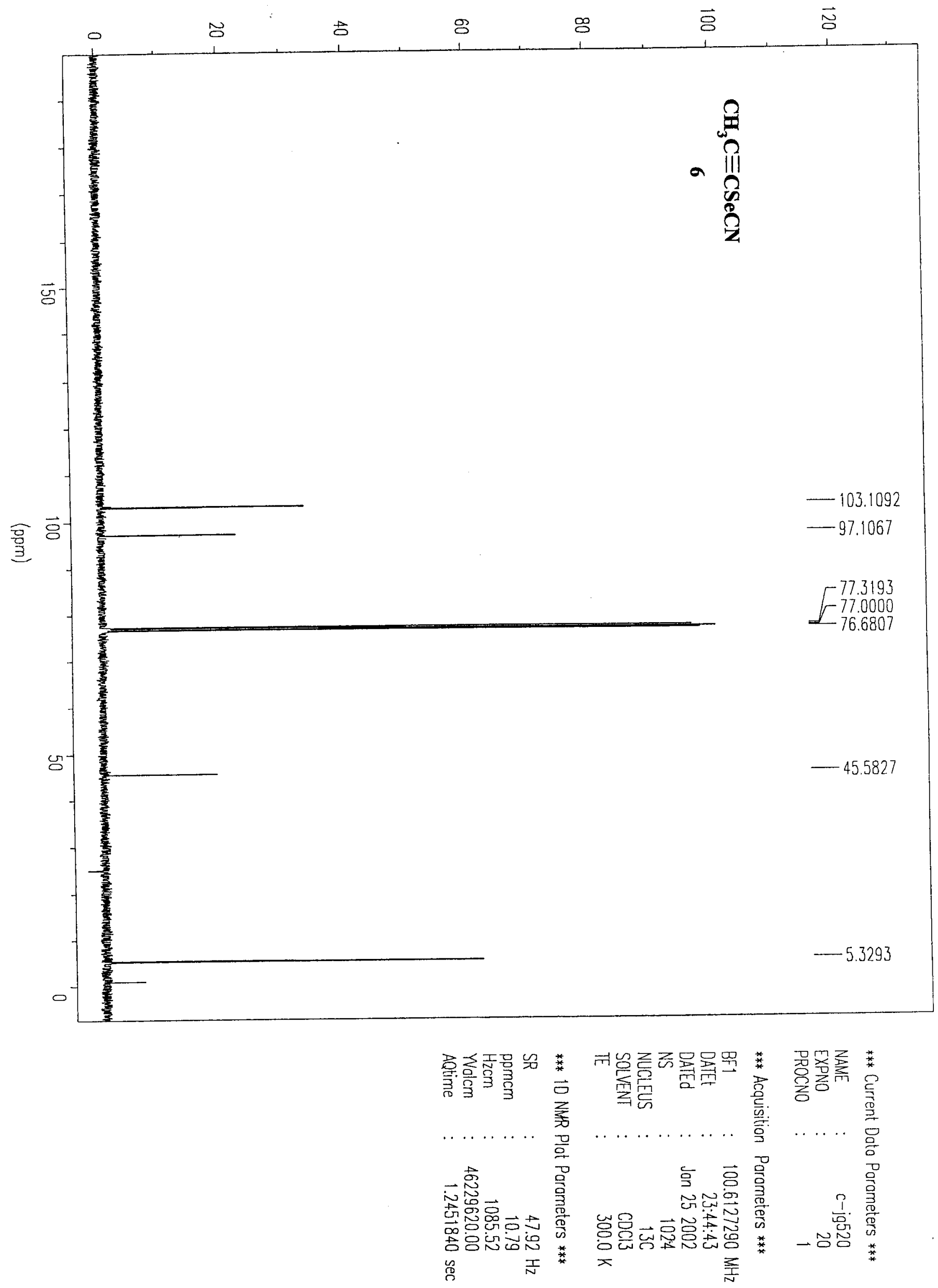

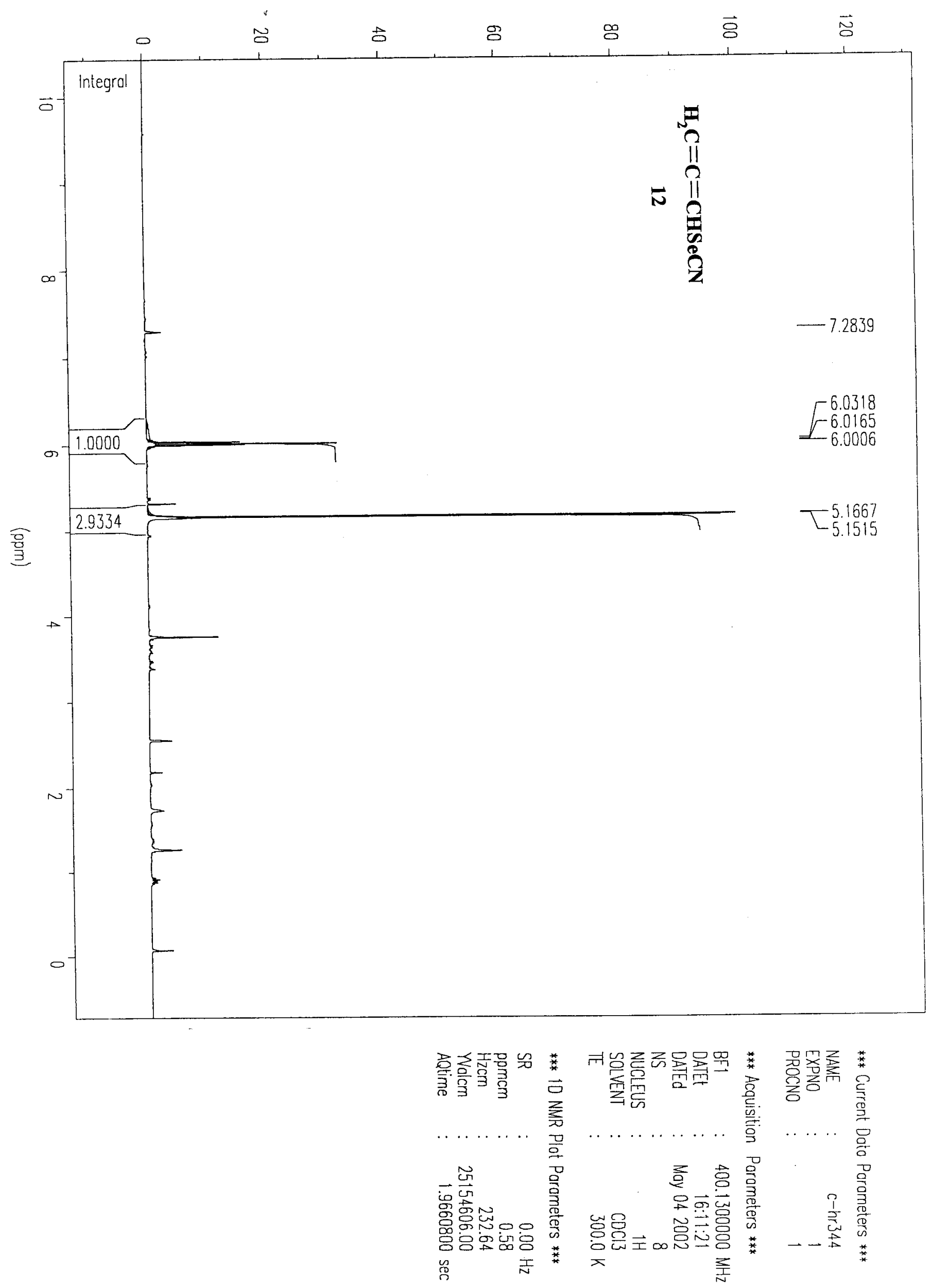


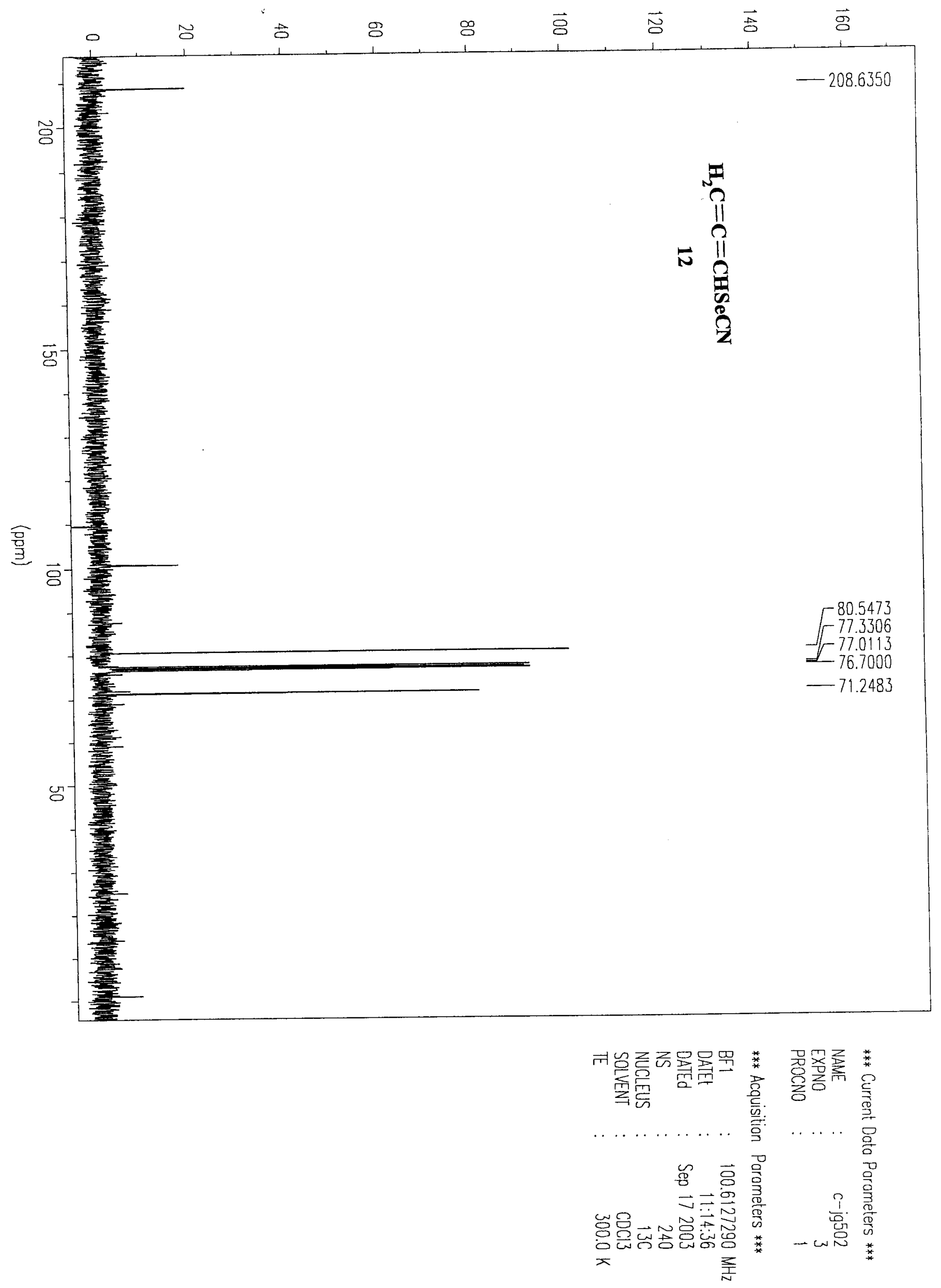



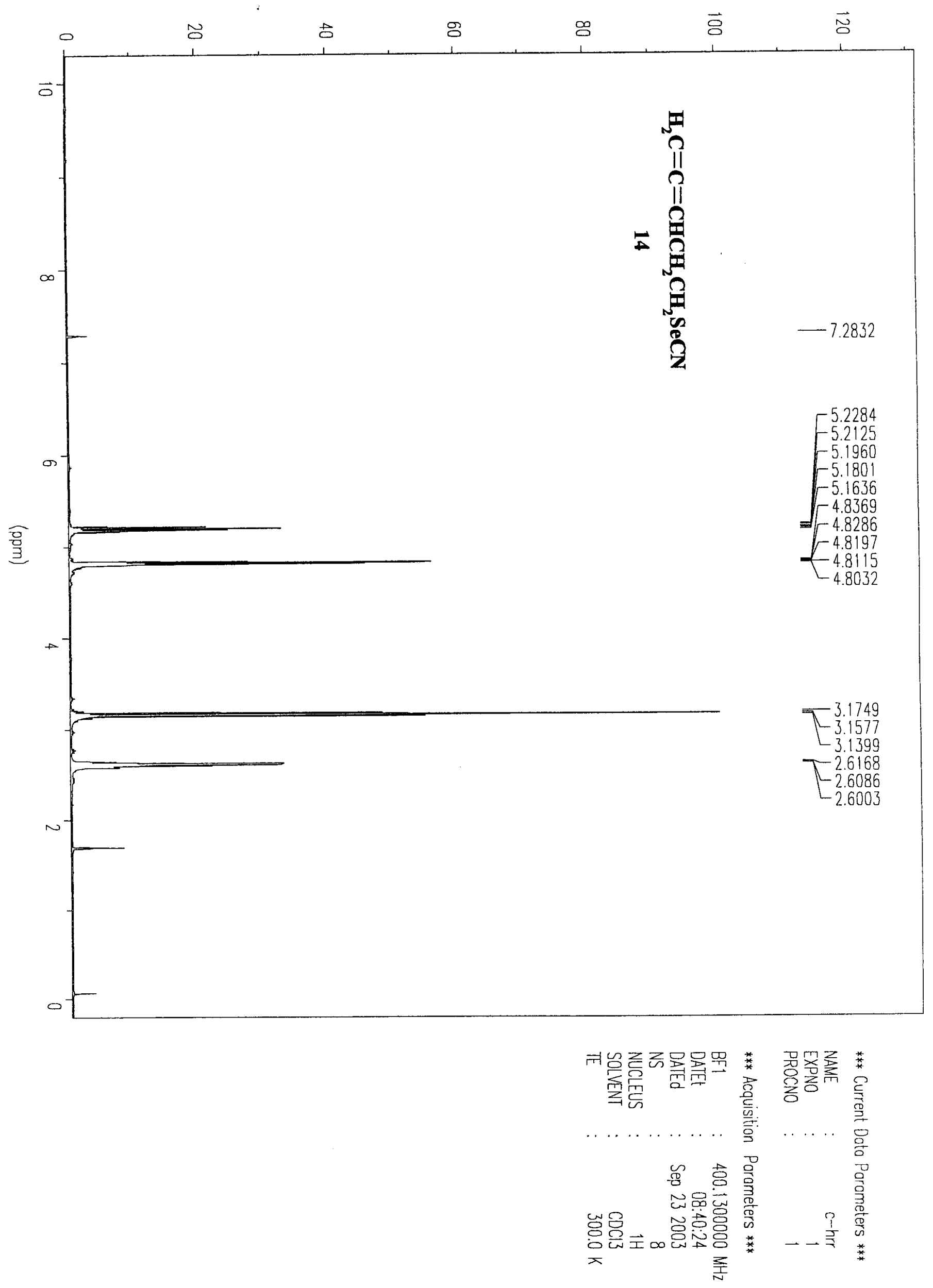

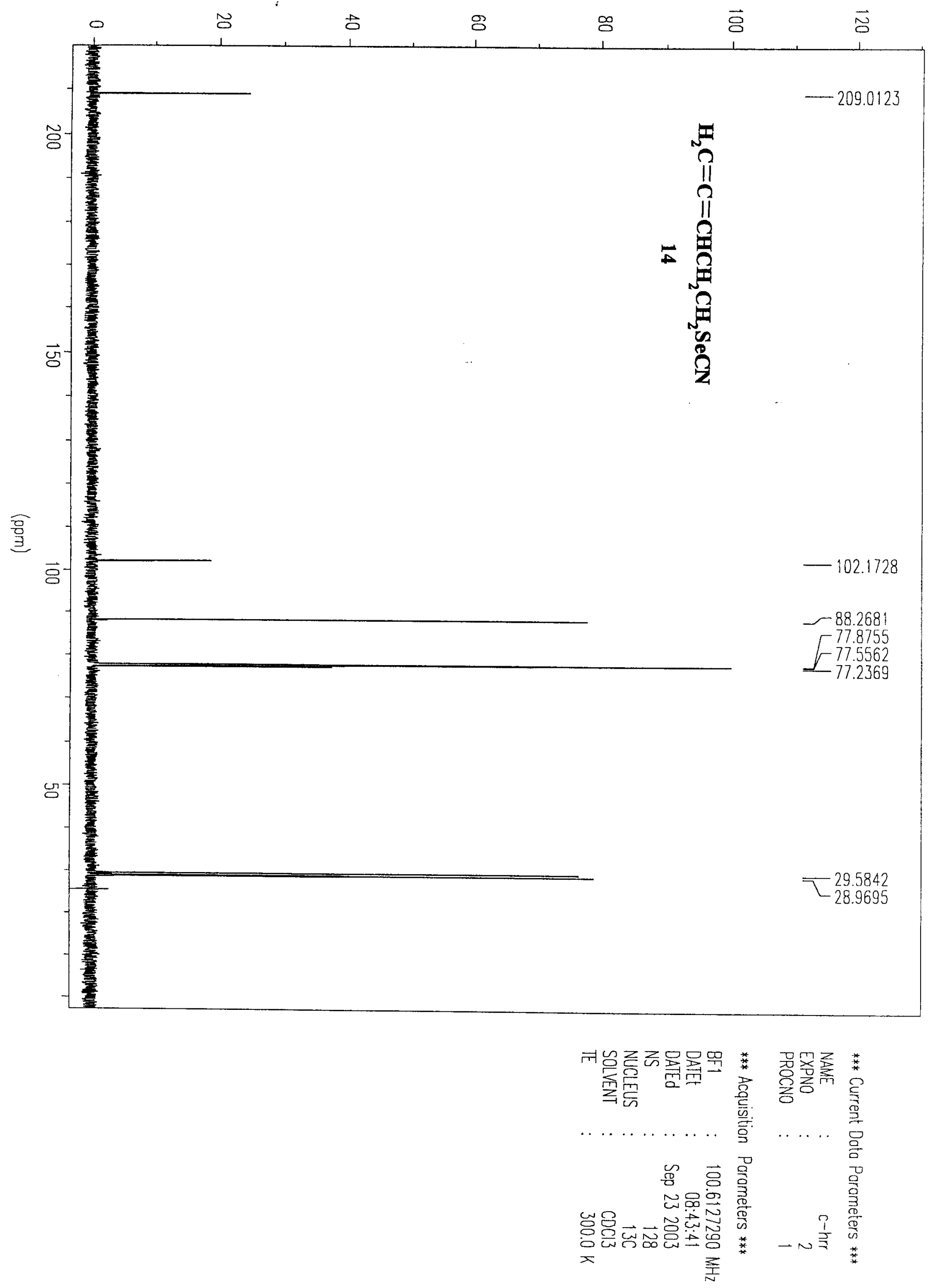


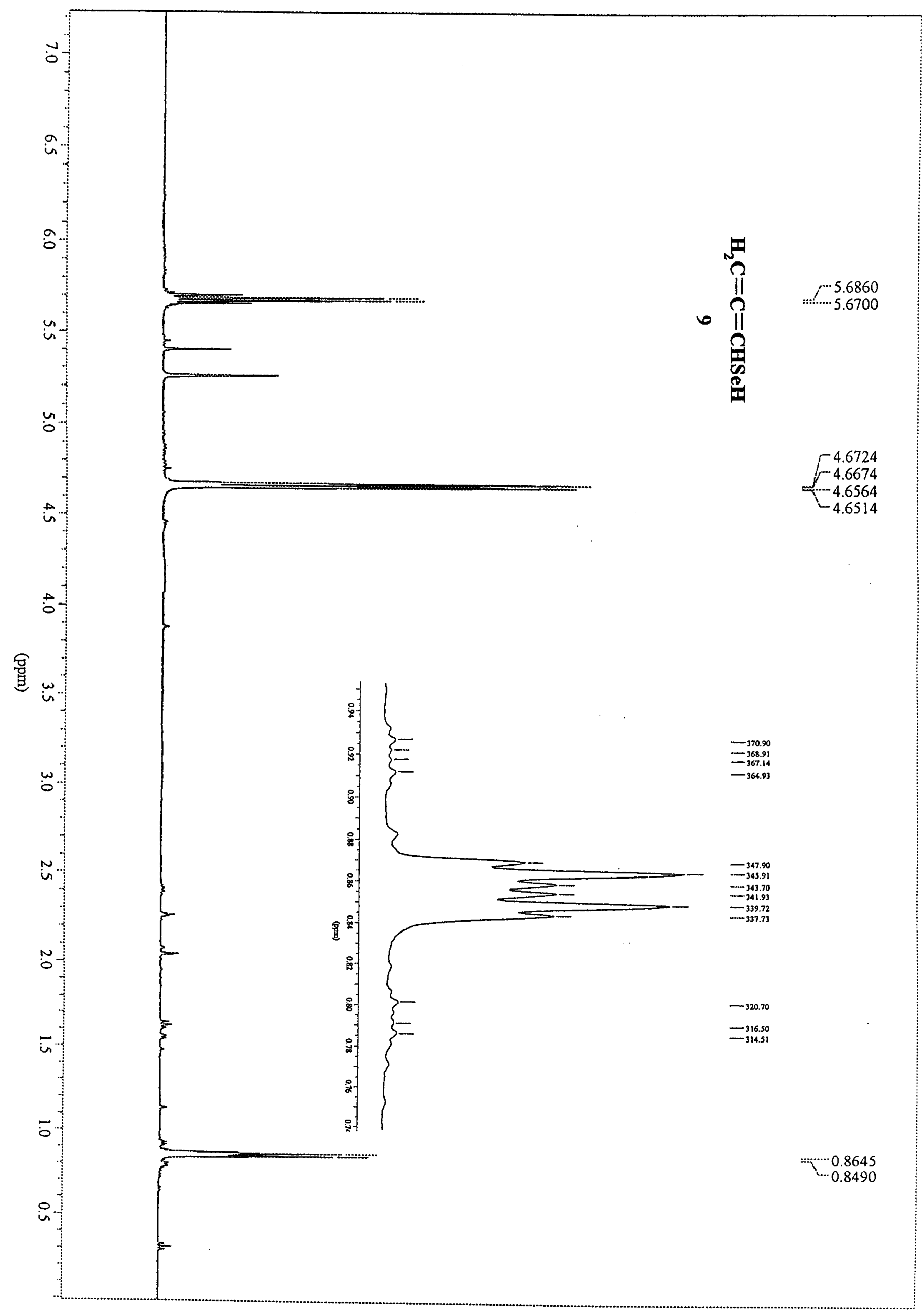



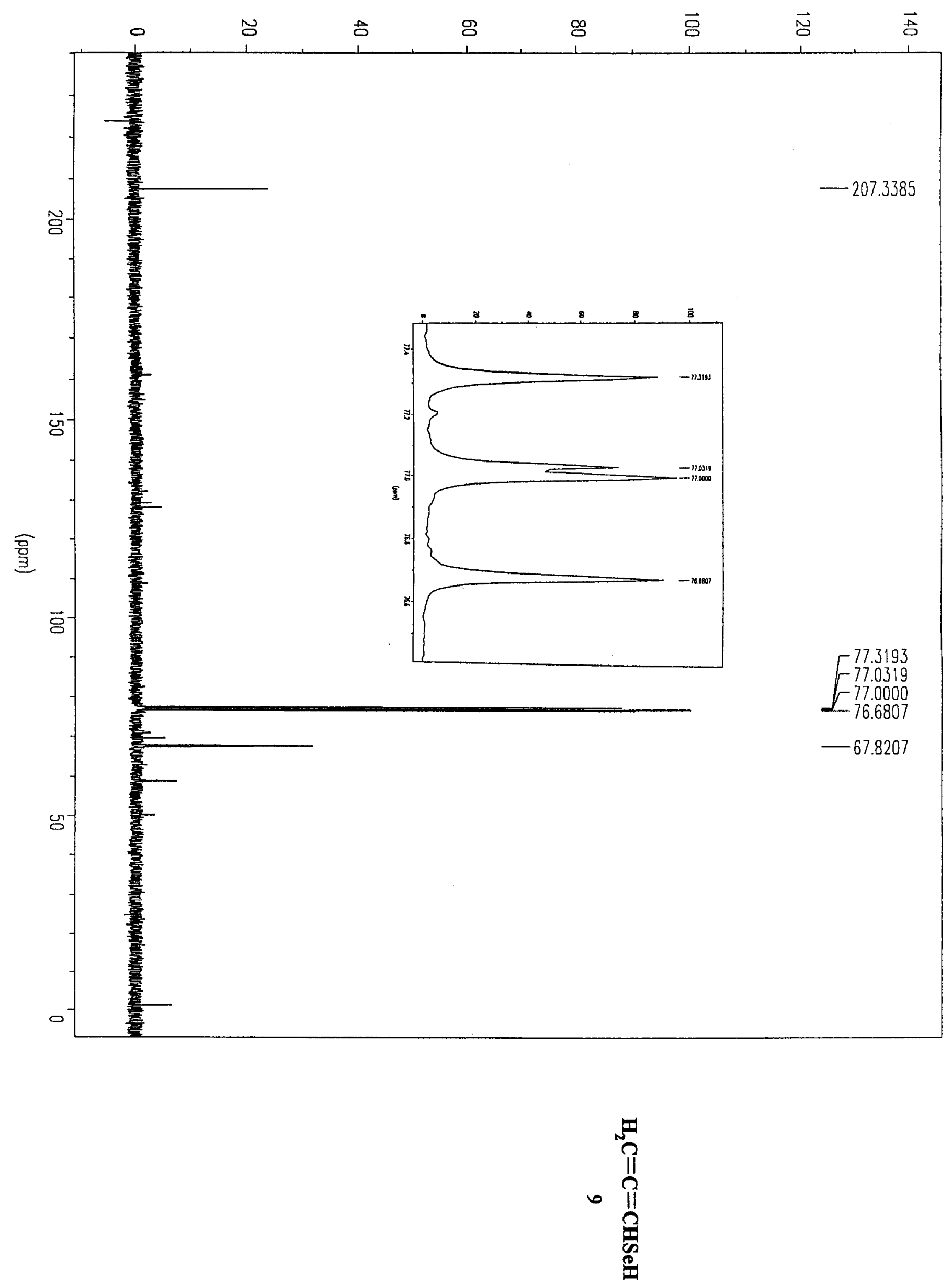

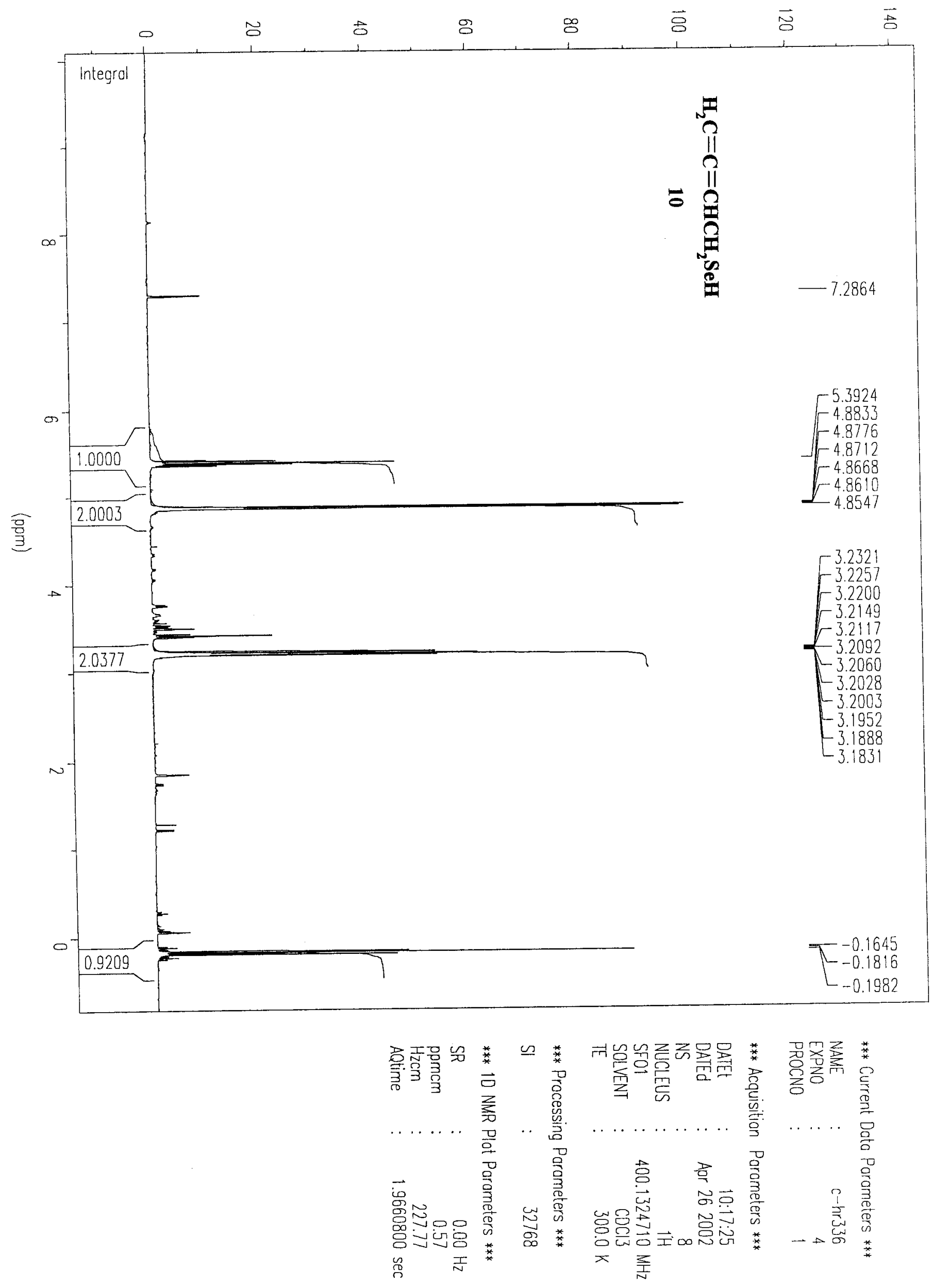

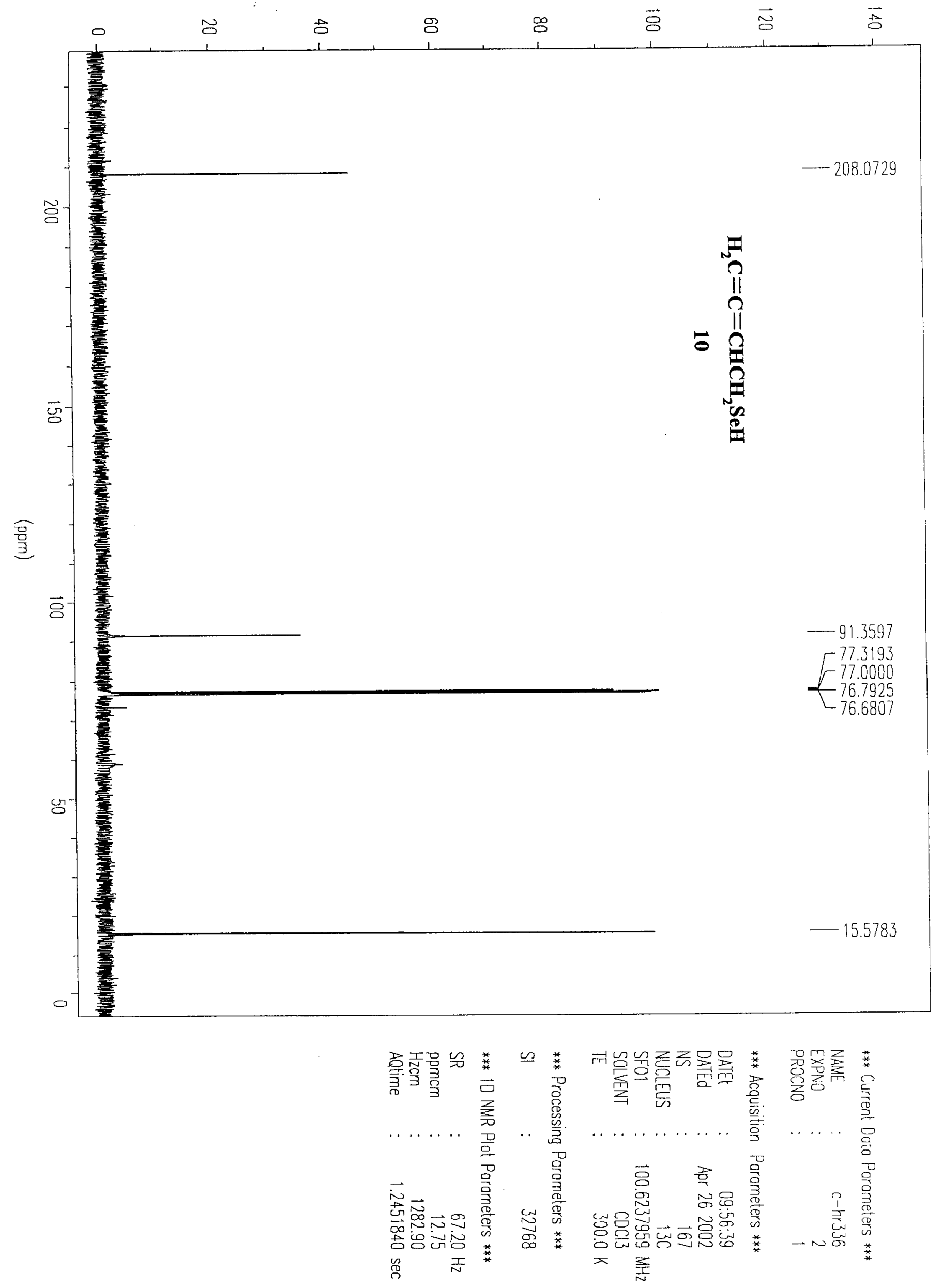


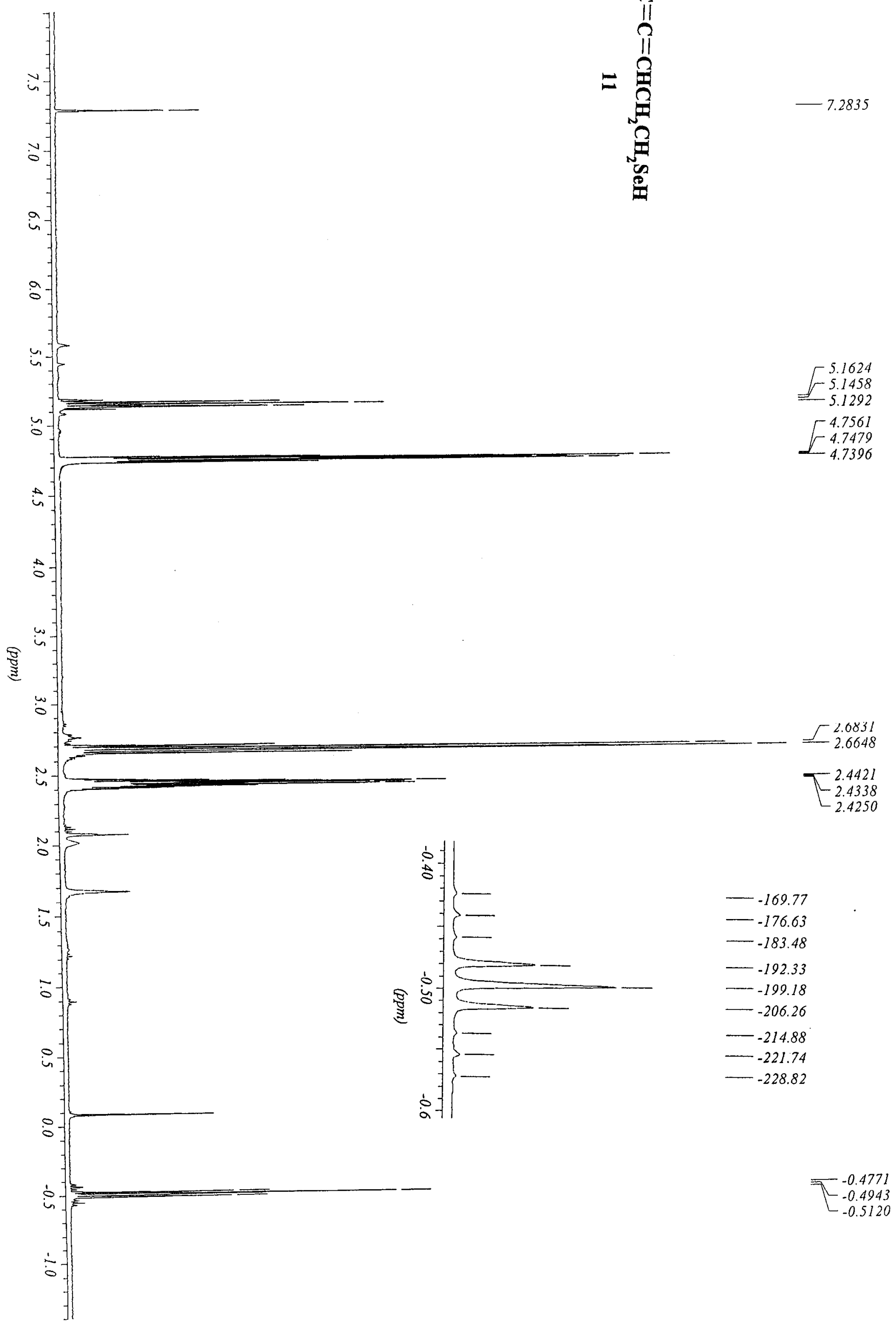




$$
\text { L }
$$

